Cahiers $d u$ MONDE RUSSE

\section{Cahiers du monde russe}

Russie - Empire russe - Union soviétique et États indépendants

$62 / 4 \mid 2021$

Varia

\title{
Lesley CHAMBERLAIN, Ministry of Darkness. How Sergei Uvarov Created Conservative Modern Russia
}

\section{Михаил Велижев}

\section{(2) OpenEdition}

\section{Journals}

Édition électronique

URL : https://journals.openedition.org/monderusse/12774

DOI : 10.4000/monderusse. 12774

ISSN : $1777-5388$

\section{Éditeur}

Éditions de l'EHESS

\section{Édition imprimée}

Date de publication : 1 décembre 2021

Pagination : 689-696

ISBN : 978-2-7132-2895-7

ISSN : $1252-6576$

Référence électronique

Михаил Велижев, «Lesley CHAMBERLAIN, Ministry of Darkness. How Sergei Uvarov Created Conservative Modern Russia», Cahiers du monde russe [Онлайн], 62/4 | 2021, Выложить онлайн 01 décembre 2021, Наводить справки в 03 septembre 2022. URL: http://journals.openedition.org/ monderusse/12774 ; DOI: https://doi.org/10.4000/monderusse.12774

Ce document a été généré automatiquement le 3 septembre 2022.

All rights reserved 


\title{
Lesley CHAMBERLAIN, Ministry of Darkness. How Sergei Uvarov Created Conservative Modern Russia
}

\author{
Михаил Велижев
}

\section{RÉFÉRENCE}

Lesley CHAMBERLAIN, Ministry of Darkness. How Sergei Uvarov Created Conservative Modern Russia, London - New York : Bloomsbury Academic, 2020, 328 p.

1 ВЫХОД В СВЕТ НОВОЙ БИОГРАФИИ С.С. УВАРОВА, ОДНОЙ КЛЮЧЕВЫХ ФИГУР РУССКОЙ ИНТЕЛЛЕКТУАЛЬНОЙ ИСТОРИИ ХІХ В., - СОБЫТИЕ, БЕЗУСЛОВНО, ЗНАЧИМОЕ. КНИГА ЛЕСЛИ ЧЕМБЕРЛЕН, ИЗВЕСТНОЙ БРИТАНСКОЙ СПЕЦИАЛИСТКИ ПО РОССИИ, ПИСАТЕЛЬНИЦЫ И ЖУРНАЛИСТКИ, ЗАСЛУЖИВАЕТ САМЫХ ЛЕСТНЫХ СЛОВ.

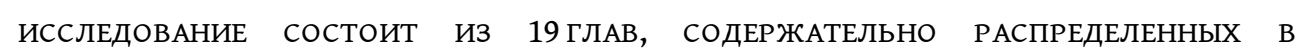
СООТВЕТСТВИИ С ХРОНОЛОГИЧЕСКИМ ПРИНЦИПОМ: ПЕРВАЯ ПОЛОВИНА РАБОТЫ ПОСВЯЩЕНА ДЕЯТЕЛЬНОСТИ УВАРОВА ДО 1825 Г., ВТОРАЯ - ЕГО ЗАНЯТИЯМ ПОСЛЕ ВОЦАРЕНИЯ ИМПЕРАТОРА НИКОЛАЯ І. МОНОГРАФИЮ ВЕНЧАЕТ ПРИЛОЖЕНИЕ, В КОТОРОМ АВТОР ДОКАЗЫВАЕТ, ЧТО УВАРОВ МОГ БЫТЬ ПРОТОТИПОМ ОДНОГО ИЗ ГЕРОЕВ РОМАНА ДЖ. КОНРАДА «НА ВЗГЛЯД ЗАПАДА» (1911). ИСТОЧНИКАМИ ЧЕМБЕРЛЕН СЛУЖАТ ПРЕЖДЕ ВСЕГО ОПУБЛИКОВАННЫЕ ПРОИЗВЕДЕНИЯ ЗНАМЕНИТОГО МИНИСТРА НАРОДНОГО ПРОСВЕЩЕНИЯ. АВТОР ПОДРОБНО АНАЛИЗИРУЕТ УВАРОВСКИЕ СОЧИНЕНИЯ, РАССМАТРИВАЯ ИХ В ДВОЙНОЙ ПРОЕКЦИИ: ВО-ПЕРВЫХ, В КОНТЕКСТЕ Weltanschauung ГЕРОЯ, ВО-ВТОРЫХ, В БОЛЕЕ ШИРОКОЙ ПЕРСПЕКТИВЕ РУССКОЙ ИСТОРИИ ПЕРВОЙ ПОЛОВИНЫ ХІХ В. КАК КАЖЕТСЯ, КНИГА ПРЕЖДЕ ВСЕГО РАССЧИТАНА НА ЧИТАТЕЛЯ, МАЛО ЗНАКОМОГО С ЖИЗНЬЮ И РЕПУТАЦИЕЙ УВАРОВА: АВТОР ДАЕТ ПАНОРАМНЫЙ ОБЗОР ВСЕХ ПЕРИОДОВ БОГАТОЙ СОБЫТИЯМИ БИОГРАФИИ ЧИНОВНИКА И, КАК СЛЕДСТВИЕ, НЕ ИМЕЕТ ВОЗМОЖНОСТИ ВХОДИТЬ В ДЕТАЛИ КАЖДОГО ИЗ ЕЕ КОНКРЕТНЫХ эПИзОдов. 
НА НАШ ВЗГЛЯД, САМОЕ ИНТЕРЕСНОЕ В ИССЛЕДОВАНИИ - ЭТО КОНЦЕПТУАЛЬНАЯ РАМКА, В КОТОРУЮ ЧЕМБЕРЛЕН ПОМЕЩАЕТ ФИГУРУ УВАРОВА. И ЗДЕСЬ НАМ ЕСТЬ, С ЧЕМ СРАВНИВАТЬ: К НАСТОЯЩЕМУ МОМЕНТУ ВЫШЛО ДОСТАТОЧНО БОЛЬШОЕ ЧИСЛО ИНТЕРЕСНЫХ РАБОТ, ПОСВЯЩЕННЫХ ТРУДАМ МИНИСТРА НАРОДНОГО ПРОСВЕЩЕНИЯ И МНОГОЛЕТНЕГО ПРЕЗИДЕНТА ИМПЕРАТОРСКОЙ АКАДЕМИИ НАУК ${ }^{1}$ ТАК, УВАРОВ ЦИНТИИ ВИТТЕКЕР ${ }^{2}$ - УСПЕШНЫЙ УЧЕНЫЙ И ИНТЕРЕСНЫЙ МЫСЛИТЕЛЬ, СОЗДАТЕЛЬ НОВОЙ ИМПЕРСКОЙ СИСТЕМЫ ВЫСШЕГО ОБРАЗОВАНИЯ, ОДИН ИЗ КЛЮЧЕВЫХ РУССКИХ ГОСУДАРСТВЕННЫХ ДЕЯТЕЛЕЙ ХІХ В., ЧЕЛОВЕК СО СЛОЖНЫМ ХАРАКТЕРОМ И ДУРНОЙ РЕПУТАЦИЕЙ, ВПРОЧЕМ, ЗНАВШИЙ СЕБЕ ЦЕНУ И НЕ ОБРАЩАВШИЙ ВНИМАНИЯ НА НЕДОБРОЖЕЛАТЕЛЕЙ. УВАРОВ РИЧАРДА ПАЙПСА ${ }^{3}-$ СКОРЕЕ ЭФФЕКТИВНЫЙ АДМИНИСТРАТОР, НЕЖЕЛИ УЧЕНЫЙ ИЛИ СОЧИНИТЕЛЬ, ЧЬЕ НЕПОМЕРНОЕ ТЩЕСЛАВИЕ КОМПЕНСИРУЕТСЯ ЕГО ДЕЙСТВИТЕЛЬНЫМИ ЗАСЛУГАМИ ПЕРЕД РУССКОЙ КУЛЬТУРОЙ. УВАРОВ АНДРЕЯ ЗОРИНА ${ }^{4}$ - УМНЫЙ И ТОНКИЙ ИДЕОЛОГ, СФОРМУЛИРОВАВШИЙ ОСНОВОПОЛАГАЮЩИЙ ПРИНЦИП ОФИЦИАЛЬНОГО НАЦИОНАЛИЗМА САМОДЕРЖАВИЕ ПРАВОСЛАВИЕ - НАРОДНОСТЬ». ЕМУ УДАЛОСЬ ПРЕДЛОЖИТЬ НИКОЛАЮ І СИСТЕМУ ВЗГЛЯДОВ, СПОСОБНУЮ ПОДДЕРЖАТЬ УСТОЙЧИВОСТЬ ПОЛИТИЧЕСКОГО ПОРЯДКА В КОНТЕКСТЕ ОСОЗНАННОЙ ПОЛИТИКИ ТОРМОЖЕНИЯ РАДИКАЛЬНЫХ ИЗМЕНЕНИЙ В СТРАНЕ, КОГДА ИМПЕРАТОР НЕ РЕШИЛСЯ ПРИСТУПИТЬ К РЕФОРМАМ И ОТЛОЖИЛ ИХ РЕАЛИЗАЦИЮ. УВАРОВ МАРИИ МАЙОФИС ${ }^{5}$ - ОДИН ИЗ САМЫХ ИНТЕРЕСНЫХ РУССКИХ

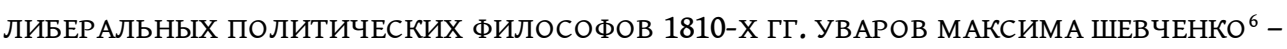
«БЛАГОРОДНЫЙ КОНСЕРВАТОР», ПРИДЕРЖИВАВШИЙСЯ ПРИНЦИПОВ ПРАВОСЛАВИЯ И САМОДЕРЖАВИЯ. БУДУЧИ ОХРАНИТЕЛЕМ, ОН ТЕМ НЕ МЕНЕЕ ПОНИМАЛ, ЧТО УСПЕХИ В ОБЛАСТИ НАУКИ И ОБРАЗОВАНИЯ НЕИЗМЕННО ПРИВЕДУТ К ГРАЖДАНСКОЙ АКТИВНОСТИ ПОДДАННЫХ, КОТОРУЮ СЛЕДОВАЛО НАПРАВЛЯТЬ В ОПРЕДЕЛЕННОЕ РУСЛО, А НЕ БЕЗДУМНО ГАСИТЬ.

УВАРОВ ЛЕСЛИ ЧЕМБЕРЛЕН - ПРЕЖДЕ ВСЕГО, ЧЕЛОВЕК С РАЗДВОЕННЫМ СОЗНАНИЕМ И МИРОВОЗЗРЕНИЕМ, НА ВСЕМ ПРОТЯЖЕНИИ ЖИЗНИ РАЗРЫВАВШИЙСЯ МЕЖДУ ИДЕАЛОМ И РЕАЛЬНОСТЬЮ, ЛИБЕРАЛИЗМОМ И КОНСЕРВАТИЗМОМ, КУЛЬТУРОЙ XVIII И ХІХ СТОЛЕТИЯ, ПУБЛИЧНЫМ И ЧАСТНЫМ ПРОСТРАНСТВОМ. ЗНАЧИТЕЛЬНОЕ МЕСТО В РАБОТЕ АВТОР УДЕЛЯЕТ РЕКОНСТРУКЦИИ ХАРАКТЕРА И ПСИХОЛОГИИ ГЕРОЯ. АМБИВАЛЕНТНОСТЬ ВО ВЗГЛЯДАХ НА ОКРУЖАЮЩИЙ МИР ОКАЗАЛАСЬ СВОЙСТВЕННА УВАРОВУ С САМОЙ ЮНОСТИ, КОГДА ВО ВТОРОЙ ПОЛОВИНЕ 1800-Х ГГ. ОН ВЫПОЛНЯЛ ДИПЛОМАТИЧЕСКИЕ ПОРУЧЕНИЯ В ВЕНЕ. ЧЕМБЕРЛЕН ОТМЕЧАЕТ, ЧТО МОЛОДОГО ЧИНОВНИКА, В ЧАСТНОСТИ, ВДОХНОВЛЯЛИ ИДЕИ ПРУССКОГО РЕФОРМАТОРА БАРОНА ФОН ШТЕЙНА, СЧИТАВШЕГО ПРОСВЕЩЕНИЕ ШИРОКИХ СЛОЕВ НАСЕЛЕНИЯ НЕОБХОДИМЫМ УСЛОВИЕМ ПОЛИТИЧЕСКОЙ ЛИБЕРАЛИЗАЦИИ. ОДНАКО В ТО ЖЕ ВРЕМЯ УВАРОВ ПОЛАГАЛ, ЧТО РАСПРОСТРАНЕНИЕ ОБРАЗОВАНИЯ СПОСОБНО ПРИВЕСТИ К РОСТУ РЕСПУБЛИКАНСКИХ НАСТРОЕНИЙ, ЧТО НЕ ВЫЗЫВАЛО У НЕГО НИКАКОГО ЭНТУЗИАЗМА.

СТАВ В 1810 Г. ПОПЕЧИТЕЛЕМ САНКТ-ПЕТЕРБУРГСКОГО УЧЕБНОГО ОКРУГА, УВАРОВ СТРЕМИЛСЯ ПРИМИРИТЬ САМОДЕРЖАВИЕ И ПРОГРЕСС, СОЧЕТАЯ ПРАКТИЦИЗМ И ИДЕАЛИЗМ В ИСПОЛНЕНИИ СЛУЖЕБНЫХ ОБЯЗАННОСТЕЙ. ЧЕМБЕРЛЕН ОПРЕДЕЛЯЕТ УВАРОВА ТАК: «РУССКИЙ КОНСЕРВАТИВНЫЙ РОМАНТИК, ВДОХНОВЛЯВШИЙСЯ ЗАПАДОМ (foreign-inspired), ЛИБЕРАЛЬНЫЙ В СВОЕМ ПОДХОДЕ К ОБРАЗОВАНИЮ, НО В ВЫСШЕЙ СТЕПЕНИ ОСТОРОЖНЫЙ В ВОПРОСАХ ПОЛИТИЧЕСКИХ РЕФОРМ» (С. 49). СТРАСТНАЯ ПРИВЕРЖЕННОСТЬ ЕВРОПЕЙСКОЙ ИНТЕЛЛЕКТУАЛЬНОЙ КУЛЬТУРЕ В СОЗНАНИИ 
УВАРОВА ПРЕЖДЕ ВСЕГО СВЯЗЫВАЛАСЬ НЕ С СОЦИАЛЬНЫМ ПРОГРЕССОМ, А С ЭСТЕТИЗАЦИЕЙ ДЕЙСТВИТЕЛЬНОСТИ, НЕ ВСТУПАВШЕЙ В ПРОТИВОРЕЧИЕ С АБСОЛЮТИСТСКИМ ТИПОМ ПОЛИТИЧЕСКОГО ПРАВЛЕНИЯ. ПОДОБНАЯ ПОЗИЦИЯ БУДЕТ ХАРАКТЕРНА ДЛЯ ГЕРОЯ КНИГИ ВО ВСЕ ЭПОХИ ЕГО ПРОФЕССИОНАЛЬНОЙ ДЕЯТЕЛЬНОСТИ И РОВНО В ЭТОМ СМЫСЛЕ ОН ОСТАНЕТСЯ «СТАРОРЕЖИМНЫМ» ЧЕЛОВЕКОМ XVIII В. В ЭПОХУ СТРЕМИТЕЛЬНЫХ ПЕРЕМЕН ХІХ СТОЛЕТИЯ.

5 ТА ЖЕ РАЗДВОЕННОСТЬ ПРИСУЩА И ВЗГЛЯДАМ УВАРОВА НА РОССИЮ. ПРИВОДЯ СВИДЕТЕЛЬСТВА СОВРЕМЕННИКОВ И ИСТОРИКОВ, ЧЕМБЕРЛЕН СПРАВЕДЛИВО ОТМЕЧАЕТ, ЧТО СТОЛИЧНЫЙ ПОПЕЧИТЕЛЬ СТРАННЫМ ОБРАЗОМ МАЛО ИНТЕРЕСОВАЛСЯ СОБСТВЕННЫМ ОТЕЧЕСТВОМ. ТЕМ НЕ МЕНЕЕ ЕГО ВПОЛНЕ МОЖНО СЧИТАТЬ «ПАТРИОТОМ» С ТОЙ ОГОВОРКОЙ, ЧТО ЛЮБОВЬ УВАРОВА К СТРАНЕ ГЛАВНЫМ ОБРАЗОМ ЗАКЛЮЧАЛАСЬ В СТРЕМЛЕНИИ УТВЕРДИТЬ ГОСУДАРСТВЕННЫЙ ПРЕСТИЖ, В ТО ВРЕМЯ КАК КУЛЬТУРНАЯ ИДЕНТИЧНОСТЬ ИМПЕРИИ ЧИНОВНИКА ИНТЕРЕСОВАЛА КУДА МЕНЬШЕ. СТРАСТНО ЖЕЛАВШИЙ ЖИТЬ ЕВРОПЕЙЦЕМ В РОССИИ, УВАРОВ СТАЛКИВАЛСЯ С НЕРАЗРЕШИМОЙ ПРОБЛЕМОЙ - ГЛУБОКИМ РАЗРЫВОМ МЕЖДУ ДВУМЯ ПРОСТРАНСТВАМИ, УСУГУБЛЯВШИМСЯ НЕЖЕЛАНИЕМ ВЛАСТЕЙ РАЗНЫХ УРОВНЕЙ СЛЕДОВАТЬ ПРИНЦИПАМ СПРАВЕДЛИВОСТИ И ПОРЯДКА, КУЛЬТУРНЫМ ИЗОЛЯЦИОНИЗМОМ, ВОЗНИКШЕМ В 1812 Г., И ОТКРОВЕННОЙ ВРАЖДЕБНОСТЬЮ ЗАПАДНОМУ ПРОСВЕЩЕНИЮ, В ОСОБЕННОСТИ ПРОЯВИВШЕЙСЯ В 1821 Г. ВО ВРЕМЯ ИСТОРИИ С УХОДОМ УВАРОВА С ПОСТА ПОПЕЧИТЕЛЯ ВСЛЕДСТВИЕ ПРЕСЛЕДОВАНИЙ М.Л. МАГНИЦКОГО И Д.П. РУНИЧА.

6 В НИКОЛАЕВСКОЕ ЦАРСТВОВАНИЕ УВАРОВ ОТЧАСТИ ПРИМИРИЛСЯ С ОКРУЖАВШЕЙ ЕГО ДЕЙСТВИТЕЛЬНОСТЬЮ, СДЕЛАВ БЛЕСТЯЩУЮ КАРЬЕРУ И СТАВ ГЛАВНЫМ ТЕОРЕТИКОМ ОФИЦИАЛЬНОГО ИМПЕРСКОГО НАЦИОНАЛИЗМА. ВПРОЧЕМ, И ЗДЕСЬ ЕГО ПОЛОЖЕНИЕ ПРЕДСТАВЛЯЕТСЯ ВЕСЬМА ДВОЙСТВЕННЫМ. НАЧАТЬ С ТОГО, ЧТО МИНИСТР ПОЖЕРТВОВАЛ СОБСТВЕННОЙ РЕПУТАЦИЕЙ РАДИ УДОВЛЕТВОРЕНИЯ ЛИЧНОЙ АМБИЦИИ И, КАК ЕМУ КАЗАЛОСЬ, РАДИ ГОСУДАРСТВЕННОГО БЛАГА: СОВРЕМЕННИКИ (СРЕДИ КОТОРЫХ СЛЕДУЕТ ОСОБО ОТМЕТИТЬ ПУШКИНА) ПОРИЦАЛИ УВАРОВА ЗА НЕПОМЕРНОЕ ТЩЕСЛАВИЕ, ПОДОБОСТРАСТИЕ К НАЧАЛЬСТВУ, ЖАДНОСТЬ, МСТИТЕЛЬНОСТЬ, НАДМЕННОСТЬ И ДРУГИЕ ПОРОКИ. ИСКРЕННИЙ СТОРОННИК РАСПРОСТРАНЕНИЯ ЕВРОПЕЙСКОГО ОБРАЗОВАНИЯ, ОН ВОШЕЛ В ИСТОРИЮ КАК РЕАКЦИОНЕР И МИНИСТР НАРОДНОГО «ПОМРАЧЕНИЯ», ПРИ ТОМ ЧТО ЕГО УПРАВЛЕНИЕ ВЕДОМСТВОМ ПРИНЕСЛО РУССКОЙ НАУКЕ МНОГО ПОЛЬЗЫ. В РЕЗУЛЬТАТЕ, КАК НЕОДНОКРАТНО ПОДЧЕРКИВАЕТ ЧЕМБЕРЛЕН, В ПУБЛИЧНОЙ ЖИЗНИ УВАРОВЧИНОВНИК БЫЛ ОДИНОК И ОКРУЖЕН НЕДОБРОЖЕЛАТЕЛЯМИ. СИТУАЦИЯ МЕНЯЛАСЬ К ЛУЧШЕМУ ЛИШЬ В ЧАСТНОМ ПРОСТРАНСТВЕ - В ОСОБЕННОСТИ, В ПОДМОСКОВНОЙ УСАДЬБЕ ПОРЕЧЬЕ, УСТРОЕННОЙ СОГЛАСНО ЭСТЕТИЧЕСКИМ ВКУСАМ И ПРИСТРАСТИЯМ ВЛАДЕЛЬЦА, ГДЕ СОБИРАЛОСЬ ИЗБРАННОЕ ОБЩЕСТВО ДРУЗЕЙ И ПОЧИТАТЕЛЕЙ УВАРОВА.

7 ДЕЯТЕЛЬНОСТЬ УВАРОВА-ИДЕОЛОГА ТАКЖЕ ОПИСЫВАЕТСЯ «РАЗДВОЕННОГО СОЗНАНИЯ» ГЕРОЯ. СОЗДАТЕЛЬ ВЛИЯТЕЛЬНОЙ ПОЛИТИКОКОНФЕССИОНАЛЬНОЙ ДОКТРИНЫ, ОН ТЕМ НЕ МЕНЕЕ НЕ ОТЛИЧАЛСЯ РЕЛИГИОЗНОСТЬЮ (НЕСЛУЧАЙНО В ЗАПИСКАХ, ОБРАЩЕННЫХ К НИКОЛАЮ I, УВАРОВ НАЗЫВАЛ ПРАВОСЛАВИЕ «НАЦИОНАЛЬНОЙ РЕЛИГИЕЙ», УКАЗЫВАЯ НА ФУНКЦИЮ, А НЕ СУЩНОСТЬ ВОСТОЧНОЙ ВЕТВИ ХРИСТИАНСТВА) И ДАЖЕ ЗАСЛУЖИЛ УПРЕКИ В АТЕИЗМЕ. ОН ПОПРЕЖНЕМУ ЗАЩИЩАЛ ЕВРОПЕЙСКОЕ ПРОСВЕЩЕНИЕ, ОДНАКО ПРЕБЫВАЛ В 
УВЕРЕННОСТИ, ЧТО К ЗАПАДНЫМ ИНТЕЛЛЕКТУАЛЬНЫМ ЦЕННОСТЯМ РОССИЯ ИДЕТ «ОСОБЫМ», НЕ-ЗАПАДНЫМ ПУТЕМ. В НИКОЛАЕВСКУЮ ЭПОХУ ВОЗНИКАЕТ НОВАЯ ИДЕОЛОГИЯ, КОТОРАЯ ВПОСЛЕДСТВИИ ПРИВЕДЕТ К ВОЗНИКНОВЕНИЮ «ИНТЕЛЛИГЕНЦИИ», КОГДА ГЛАВНОЙ ЗАДАЧЕЙ ПИСАТЕЛЯ, УЧЕНОГО ИЛИ КРИТИКА СТАНЕТ ПРОСВЕЩЕНИЕ НИЗШИХ СЛОЕВ НАСЕЛЕНИЯ. УВАРОВ ПОДЧЕРКНУТО СТОЯЛ ВНЕ ЭТОГО ДВИЖЕНИЯ, ПОСКОЛЬКУ ОТСТАИВАЛ ИДЕИ СОСЛОВНОГО ОБРАЗОВАНИЯ И ОГРАНИЧЕНИЯ ОБЩЕСТВЕННОЙ ДИСКУССИИ, СЛУЖИВШИЕ, ПО ЕГО МНЕНИЮ, ЗАЛОГОМ ПОЛИТИЧЕСКОЙ УСТОЙЧИВОСТИ САМОДЕРЖАВНОЙ ВЛАСТИ.

КАК СЧИТАЕТ ЧЕМБЕРЛЕН, УВАРОВ ЯВЛЯЕТ СОБОЙ ХАРАКТЕРНЫЙ ПРИМЕР ОБРАЗОВАННОГО РУССКОГО ЧИНОВНИКА, СОПРОТИВЛЯВШЕГОСЯ МОДЕРНОСТИ И НЕ ПРИНИМАВШЕГО СТРЕМИТЕЛЬНЫЕ ИЗМЕНЕНИЯ, ПРОИСХОДИВШИЕ В МИРЕ. В ЭТОЙ ПЕРСПЕКТИВЕ БИОГРАФИЯ УВАРОВА И ЕГО МИРОВОЗЗРЕНИЕ СЛУЖАТ ЭМБЛЕМОЙ БОЛЕЕ ГЛОБАЛЬНЫХ ПРОЦЕССОВ РУССКОЙ ИСТОРИИ. ПО ПРЕДПОЛОЖЕНИЮ ИССЛЕДОВАТЕЛЬНИЦЫ, В РОССИИ МЫ ДО СИХ ПОР НЕРЕДКО СТАЛКИВАЕМСЯ С ЛИДЕРАМИ, КАК БЫ СУЩЕСТВУЮЩИМИ В ДВУХ ПАРАЛЛЕЛЬНЫХ МИРАХ: ПОДОБНО УВАРОВУ, ОНИ ПРИЗНАЮТ ДОСТОИНСТВА ЗАПАДНОЙ ПОЛИТИКИ И ОБРАЗОВАНИЯ, ОДНАКО РЕШИТЕЛЬНО ОТКАЗЫВАЮТСЯ ПРИМЕНЯТЬ ИХ К СОБСТВЕННОМУ ОТЕЧЕСТВУ. В ОДНОМ КОНТЕКСТЕ ОНИ ЯВЛЯЮТСЯ ПРОГРЕССИВНЫМИ ЛИБЕРАЛАМИ, В ДРУГОМ АРХАИЧНЫМИ КОНСЕРВАТОРАМИ. ИСТОРИЧЕСКИ ОЧЕРЧЕННЫЙ ДУАЛИЗМ ВОЗНИК В ПЕРВОЙ ТРЕТИ ХІХ В. ОДНОВРЕМЕННО С АРИСТОКРАТИЧЕСКОЙ КОНЦЕПЦИЕЙ РУССКОГО Sonderweg, ЧАСТЬЮ ИНТЕЛЛЕКТУАЛЬНОЙ ПРОГРАММЫ КОТОРОГО ВЫСТУПАЛО ПРЕДСТАВЛЕНИЕ О ВЕЛИЧИИ РОССИИ, УПРАВЛЕНИЕ КОТОРОЙ ТРЕБОВАЛО СДЕРЖИВАЮЩЕГО, РЕПРЕССИВНОГО ПОДХОДА. В ИТОГЕ, ЭЛИТА ОБРАЩАЛАСЬ К ГЛУБОКО ЕЙ НЕСИМПАТИЧНЫМ ПРАКТИКАМ АДМИНИСТРИРОВАНИЯ, МОТИВИРУЯ ЭТО УНИКАЛЬНОСТЬЮ РУССКОГО ПОЛИТИЧЕСКОГО ПОРЯДКА.

9 А КАК ЖЕ ОЦЕНИВАЛ ИСТОРИЮ СВОЕЙ ЖИЗНИ САМ УВАРОВ? К СОЖАЛЕНИЮ, В РАБОТЕ ЧЕМБЕРЛЕН АНАЛИЗ ЕГО АВТОБИОГРАФИИ ОТСУТСТВУЕТ, ХОТЯ К МОМЕНТУ ВЫХОДА КНИГИ УВАРОВСКИЕ МЕМУАРЫ БЫЛИ ОПУБЛИКОВАНЫ ДВАЖДЫ: М.М.ШЕВЧЕНКО И М.А. ПОЛЯКОВОЙ ${ }^{7}$ ВОСПОМИНАНИЯ МИНИСТРА СОЗДАВАЛИСЬ УЖЕ ПОСЛЕ ТОГО, КАК ОН ОСТАВИЛ ГОСУДАРСТВЕННУЮ СЛУЖБУ И ПОСЕЛИЛСЯ В ПОРЕЧЬЕ. СОГЛАСНО ПОМЕТЕ НА ОРИГИНАЛЬНОЙ РУКОПИСИ, ТЕКСТ БЫЛ ЗАКОНЧЕН 16 ИЮНЯ 1852 Г. И ЗАТЕМ ПЕРЕДАН СЫНУ УВАРОВА АЛЕКСЕЮ. АВТОР ОТМЕЧАЛ, ЧТО АВТОБИОГРАФИЯ ЯВЛЯЕТСЯ ИСКЛЮЧИТЕЛЬНО «СЕМЕЙНЫМ» ДОКУМЕНТОМ. ОДНАКО МОЖНО ПРЕДПОЛОЖИТЬ, ЧТО ОН ПИСАЛ СВОЕ СОЧИНЕНИЕ С РАСЧЕТОМ НА БОЛЕЕ ШИРОКУЮ АУДИТОРИЮ. В ПОСЛЕДНИЕ ГОДЫ ЖИЗНИ УВАРОВ ЗАНИМАЛСЯ ПРИВЕДЕНИЕМ В ПОРЯДОК СВОЕГО АРХИВА, КОТОРЫЙ СПУСТЯ НЕКОТОРОЕ ВРЕМЯ ПОСТУПИЛ В РАСПОРЯЖЕНИЕ П.И.БАРТЕНЕВА, НАНЯТОГО А.С.УВАРОВЫМ ДЛЯ НАПИСАНИЯ ПОЛНОГО ЖИЗНЕОПИСАНИЯ ОТЦА. СИСТЕМАТИЗАЦИЯ МНОГОЧИСЛЕННЫХ ЗАПИСОК И ДОКУМЕНТОВ, ВОЗМОЖНО, ПРОИЗВОДИЛАСЬ С ПРИЦЕЛОМ НА ИСПОЛЬЗОВАНИЕ ТЕКСТОВ ПРИ СОЗДАНИИ КАНОНИЧЕСКОЙ БИОГРАФИИ, ИЗ КОТОРОЙ БАРТЕНЕВ СУМЕЛ НАПИСАТЬ ЛИШЬ ПЕРВУЮ ГЛАВУ, ТАК И ОСТАВШУЮСЯ В РУКОПИСИ. КАК БЫ ТО НИ БЫЛО, МЫ ИМЕЕМ ДЕЛО С ТЩАТЕЛЬНО ПРОДУМАННЫМ АВТОБИОГРАФИЧЕСКИМ ПОВЕСТВОВАНИЕМ, ГЛАВНАЯ ЦЕЛЬ КОТОРОГО - НЕ СТОЛЬКО ОПИСАТЬ СОБЫТИЯ ИЗ ЖИЗНИ МЕМУАРИСТА, СКОЛЬКО ИНТЕРПРЕТИРОВАТЬ ИХ В СВЕТЕ ОПРЕДЕЛЕННОЙ АВТОКОНЦЕПцИИ. 

ЗНАВШЕЙ ВЗЛЕТЫ И ПАДЕНИЯ, УВАРОВ ОПИСАЛ СВОЮ ЖИЗНЬ КАК СЕРИЮ БЕЗОГОВОРОЧНЫХ ТРИУМФОВ, ЧИСЛО КОТОРЫХ СО ВРЕМЕНЕМ РОСЛО В ГЕОМЕТРИЧЕСКОЙ ПРОГРЕССИИ, ПРИЧЕМ ТРИУМФОВ ПРЕЖДЕ ВСЕГО ПРИДВОРНЫХ. ПЕРСОНАЛЬНАЯ МИФОЛОГИЯ УВАРОВА ОПИРАЛАСЬ НА ТРИ ОСНОВАНИЯ. ВО-ПЕРВЫХ, МЕМУАРИСТ ПОДЧЕРКИВАЛ СВОЮ ОСОБУЮ СВЯЗЬ С ИМПЕРАТОРСКОЙ СЕМЬЕЙ, ВОСХОДЯЩУЮ ЕЩЕ К ВРЕМЕНАМ ЕКАТЕРИНЫ ІІ, МИЛОСТЬЮ КОТОРОЙ ПОЛЬЗОВАЛСЯ ЕГО РОДИТЕЛЬ. ВО-ВТОРЫХ, УВАРОВ ОДНОЗНАЧНО ПРЕДПОЧИТАЛ ПРИДВОРНЫЙ modus operandi ПРОФЕССИОНАЛЬНОМУ МЕРИТОКРАТИЧЕСКОМУ ПРИНЦИПУ И ПОЭТОМУ СТАВИЛ СЛУЖБУ РУССКИМ МОНАРХАМ (ПРЕЖДЕ ВСЕГО, РАЗУМЕЕТСЯ, НИКОЛАЮ ПАВЛОВИЧУ) ГОРАЗДО ВЫШЕ ВСЕХ ОСТАЛЬНЫХ СВОИХ ЗАСЛУГ, В ЧАСТНОСТИ НАУЧНЫХ. НАКОНЕЦ, В-ТРЕТЬИХ, АВТОР ВОСПОМИНАНИЙ ПОДЧЕРКИВАЛ, ЧТО ЕГО ОТНОШЕНИЯ С НИКОЛАЕМ І НОСИЛИ НЕОБЫКНОВЕННО ДОВЕРИТЕЛЬНЫЙ ХАРАКТЕР, БЛАГОДАРЯ ЧЕМУ ЕМУ УДАЛОСЬ СДЕЛАТЬ БЛЕСТЯЩУЮ МИНИСТЕРСКУЮ КАРЬЕРУ. КАЖДЫЙ ИЗ ТРЕХ ПУНКТОВ НЕТРУДНО ПОДВЕРГНУТЬ КРИТИКЕ: БЛИЗОСТЬ УВАРОВА К ЦАРСКОЙ ФАМИЛИИ НАДУМАНА, ЕГО ПРИДВОРНАЯ КАРЬЕРА, В ЦЕЛОМ, СКЛАДЫВАЛАСЬ НЕ СЛИШКОМ УДАЧНО, А НИКОЛАЙ НЕ ОТНОСИЛ СЕРГЕЯ СЕМЕНОВИЧА К ЧИСЛУ СВОИХ ФАВОРИТОВ (УВАРОВ НАХОДИЛСЯ КУДА ДАЛЬШЕ ОТ МОНАРХА, ЧЕМ, СКАЖЕМ, А.Х. БЕНКЕНДОРФ, А.Ф.ОРЛОВ, И.Ф.ПАСКЕВИЧ ИЛИ И.В. ВАСИЛЬЧИКОВ). ВПРОЧЕМ, ВАЖНА ОБЩАЯ ПЕРСПЕКТИВА, В КОТОРУЮ АВТОР ПОМЕЩАЛ СВОЮ БИОГРАФИЮ. УВАРОВ СЕРГЕЯ УВАРОВА - ЭТО ИСКЛЮЧИТЕЛЬНО УСПЕШНЫЙ ПРИДВОРНЫЙ. КАК ПРЕДСТАВЛЯЕТСЯ, ЭТА ЛИНИЯ В ИССЛЕДОВАНИИ ЧЕМБЕРЛЕН ПОЧТИ ПОЛНОСТЬЮ ОПУЩЕНА.

ИСТОРИЯ С МЕМУАРАМИ УВАРОВА ДАЕТ ПОВОД ПОГОВОРИТЬ О ДРУГИХ ИСТОЧНИКАХ НЕ УЧТЕННЫХ В РЕЦЕНЗИРУЕМОЙ МОНОГРАФИИ. ЛАКУНЫ ГЛАВНЫМ ОБРАЗОМ КАСАЮТСЯ АРХИВНЫХ МАТЕРИАЛОВ, ОТЛОЖИВШИХСЯ В УВАРОВСКОМ ФОНДЕ ОПИ ГИМ В МОСКВЕ, ЧИСЛО КОТОРЫХ ДОСТАТОЧНО ВЕЛИКО. ТАК, ВНУШИТЕЛЬНЫЙ КОРПУС ТЕКСТОВ УВАРОВА ВОСХОДИТ К 1826-1831 ГГ., КОГДА ОН ФАКТИЧЕСКИ ОКАЗАЛСЯ НА ОБОЧИНЕ СИСТЕМЫ ГОСУДАРСТВЕННОГО УПРАВЛЕНИЯ И СТРЕМИЛСЯ ВНОВЬ СТАТЬ ЕЕ ЧАСТЬЮ9. В 1828-1829 ГГ. ОН СОЗДАЛ ОБШИРНОЕ ФРАНКОЯЗЫЧНОЕ СОЧИНЕНИЕ «Е́tudes sur la Russie au XIX ${ }^{\mathrm{e}}$ siècle», АДРЕСОВАННОЕ ПРЕЖДЕ ВСЕГО НИКОЛАЮ І. РАБОТА СОСТОЯЛА ИЗ ТРЕХ КНИГ, РАЗБИТЫХ НА МНОГОЧИСЛЕННЫЕ ГЛАВЫ: «Droit politique», «Population. Agriculture. Industrie. Commerce» И «Éducation. Sciences. Arts». КРОМЕ ТОГО, В АРХИВЕ СОХРАНИЛИСЬ ОТДЕЛЬНЫЕ ПРОИЗВЕДЕНИЯ, НАПИСАННЫЕ В РАЗНЫЕ годы: «De la peine capitale en Russie», «De la servitude personelle en Russie», «Esprit social. Moeurs et coutumes», «Des rapports de l'État avec l'Église en Russie», «Politique et législation (Fragment)», «Système judiciaire», «Études sur l'Empereur Alexandre», A ТАКЖЕ СЕРИЯ РУССКОЯЗЫЧНЫХ МАТЕРИАЛОВ, ЧАСТИЧНО УЖЕ ИЗВЕСТНЫХ В ПЕЧАТИ. АНАЛОГИЧНАЯ СИТУАЦИЯ НАБЛЮДАЕТСЯ И В СЛУЧАЕ ЭПИСТОЛЯРНОГО И ЛИТЕРАТУРНОГО НАСЛЕДИЯ УВАРОВА: ФАКТИЧЕСКИ ЧЕМБЕРЛЕН УЧИТЫВАЕТ ЛИШЬ МЕНЬШУЮ ЕГО ЧАСТЬ. ДО СИХ ПОР НЕОПУБЛИКОВАННЫМИ ОСТАЮТСЯ СОТНИ ПИСЕМ ЧИНОВНИКА (В ЧАСТНОСТИ, ЕГО КРАЙНЕ ЛЮБОПЫТНАЯ КОРРЕСПОНДЕНЦИЯ, АДРЕСАТОМ КОТОРОЙ БЫЛ БЕНКЕНДОРФ) ДЕСЯТКИ СТИХОТВОРЕНИЙ И ОТДЕЛЬНЫЕ ПРОЗАИЧЕСКИЕ ОТРЫВКИ, СОХРАНИВШИЕСЯ В НЕСКОЛЬКИХ АЛЬБОМАХ И ЧЕРНОВЫХ ВАРИАНТАХ $^{10}$. БИОГРАФИЯ МИНИСТРА НАРОДНОГО ПРОСВЕЩЕНИЯ, НАПИСАННАЯ С УЧЕТОМ ВСЕХ УКАЗАННЫХ ВЫШЕ ДОКУМЕНТОВ ${ }^{11}$, МОГЛА БЫ ВЫГЛЯДЕТЬ ИНАЧЕ.

Cahiers du monde russe, $62 / 4 \mid 2021$ 
К СОЖАЛЕНИЮ, В ТЕКСТ МОНОГРАФИИ ЧЕМБЕРЛЕН ВКРАЛОСЬ БОЛЬШОЕ КОЛИЧЕСТВО ФАКТИЧЕСКИХ ОШИБОК, КОТОРЫЕ НЕСКОЛЬКО ПОРТЯТ ВПЕЧАТЛЕНИЕ ОТ ИНТЕРЕСНОЙ И СОДЕРЖАТЕЛЬНОЙ КНИГИ. ТАК, АВТОР ПУТАЕТ ЕКАТЕРИНУ ІІ И ВЕЛ. КНЯЖНУ ЕКАТЕРИНУ ПАВЛОВНУ КАК ЗАКАЗЧИКА ЗАПИСКИ КАРАМЗИНА «О ДРЕВНЕЙ И НОВОЙ РОССИИ» (С.3). ВОПРЕКИ УТВЕРЖДЕНИЮ ЧЕМБЕРЛЕН (С. 21), УВАРОВ НЕ УЧИЛСЯ В ГЕТТИНГЕНСКОМ УНИВЕРСИТЕТЕ ${ }^{12}$ (МЕЖДУ ТЕМ, ИССЛЕДОВАТЕЛЬНИЦА ДЕЛАЕТ НА ОСНОВЕ НЕВЕРНЫХ СВЕДЕНИЙ ДАЛЕКО ИДУЩИЕ ВЫВОДЫ). НА С. 57 КАРАМЗИН НАЗВАН АВТОРОМ «ДЕВЯТИТОМНОЙ» ИСТОРИИ РОССИИ, МЕЖДУ ТЕМ КАК «ИСТОРИЯ ГОСУДАРСТВА РОССИЙСКОГО НАСЧИТЫВАЛА ДВЕНАДЦАТЬ ТОМОВ. ПОЭМА ЧЕРНЕЦ» ПРИНАДЛЕЖАЛА ПЕРУ И.И. КОЗЛОВА, А НЕ В.А.ЖУКОВСКОГО (С. 105). ЖУРНАЛИСТА, ПРОЗАИКА И МЕМУАРИСТА И.И. ПАНАЕВА ЗВАЛИ НЕ ИГОРЬ (С. 149), А ИВАН. ТЕЗИС О ТОМ, ЧТО ОФИЦИАЛЬНОЕ ЗАКРЫТИЕ «МОСКОВСКОГО ТЕЛЕГРАФА» В 1834 Г. СТАЛО ПЕРВЫМ СЛУЧАЕМ ПОДОБНОГО РОДА В НИКОЛАЕВСКОЕ ЦАРСТВОВАНИЕ (С. 149), НЕВЕРЕН: В 1832Г. ПО РАСПОРЯЖЕНИЮ ИМПЕРАТОРА БЫЛА ПРЕКРАЩЕНА ДЕЯТЕЛЬНОСТЬ «ЕВРОПЕЙЦА» И.В.КИРЕЕВСКОГО. БУЛГАРИНА ЕДВА ЛИ УМЕСТНО НАЗЫВАТЬ «court scribbler» (С. 152): БУДУЧИ ПОПУЛЯРНЫМ ПИСАТЕЛЕМ, ОН ТЕМ НЕ МЕНЕЕ НЕ ИМЕЛ НИКАКОГО ОТНОШЕНИЯ К ВЫСШЕЙ ПРОСЛОЙКЕ ПЕТЕРБУРГСКОЙ АРИСТОКРАТИИ. Ж.-Ш. ДАНТЕС, УБИВШИЙ ПУШКИНА НА ДУЭЛИ, СЛУЖИЛ К КОНЦУ 1836 Г. В ЧИНЕ ПОРУЧИКА, А НЕ КАПИТАНА (С. 157). О БЕЛИНСКОМ ЧЕМБЕРЛЕН ПИШЕТ (с. 163), ЧТО ОН БЫЛ СЫНОМ СЕЛЬСКОГО СВЯЩЕННИКА И ДО КОНЦА ЖИЗНИ ОСТАВАЛСЯ ХОЛОСТЯКОМ. НА САМОМ ДЕЛЕ, К ДУХОВНОМУ СОСЛОВИЮ ПРИНАДЛЕЖАЛ ДЕД, А НЕ ОТЕЦ ИЗВЕСТНОГО КРИТИКА, А В 1843 Г. БЕЛИНСКИЙ ЖЕНИЛСЯ НА М.В. ОРЛОВОЙ. НА С. 217 И 223 С.Г. СТРОГАНОВ ИМЕНУЕТСЯ «РЕКТОРОМ МОСКОВСКОГО УНИВЕРСИТЕТА». НА САМОМ ДЕЛЕ, ОН ИСПОЛНЯЛ ДОЛЖНОСТЬ ПОПЕЧИТЕЛЯ МОСКОВСКОГО УЧЕБНОГО ОКРУГА. АЛЕКСАНДРО-НЕВСКАЯ ЛАВРА, В НЕКРОПОЛЕ КОТОРОЙ ЗАХОРОНЕН ЖУКОВСКИЙ, НАХОДИТСЯ НЕ В МОСКВЕ (С. 239), А В ПЕТЕРБУРГЕ. НАКОНЕЦ, ЧЕМБЕРЛЕН МНОГОКРАТНО ИСПОЛЬЗУЕТ ПОНЯТИЕ «ИНТЕЛЛИГЕНЦИЯ» ПРИ ОПИСАНИИ ИНТЕЛЛЕКТУАЛЬНОЙ ЖИЗНИ РОССИИ ПЕРВОЙ ПОЛОВИНЫ ХІХ В. (С. 119, 129, 132, 149, 161, 166, 215 И ДР.). НА НАШ ВЗГЛЯД, ТАКОЕ СЛОВОУПОТРЕБЛЕНИЕ, БУДУЧИ АНАХРОНИЗМОМ, СПОСОБНО СБИТЬ ЧИТАТЕЛЯ С ТОЛКУ: САМО ПОНЯТИЕ ВОЗНИКАЕТ В РУССКОМ ЯЗЫКЕ ПОЗЖЕ, УЖЕ В ЭПОХУ АЛЕКСАНДРА ІІ, А ЕГО ЗНАЧЕНИЕ ПРЕТЕРПЕЛО СО ВРЕМЕНЕМ НЕСКОЛЬКО СУЩЕСТВЕННЫХ ТРАНСФОРМАЦИЙ (ТАК, РУССКИЙ «ИНТЕЛЛИГЕНТ» ХІХ ВЕКА НЕ ПОХОЖ НА СОВЕТСКОГО ИНТЕЛЛИГЕНТА» ВТОРОЙ ПОЛОВИНЫ ХХ-ГО СТОЛЕТИЯ). НЕСМОТРЯ НА ВЫСКАЗАННЫЕ ЗАМЕЧАНИЯ, МЫ УБЕЖДЕНЫ, ЧТО КНИГА ЧЕМБЕРЛЕН - ВАЖНОЕ СОБЫТИЕ В СОВРЕМЕННОЙ РУСИСТИКЕ. МЫ НАДЕЕМСЯ, ЧТО МОНОГРАФИЯ СТИМУЛИРУЕТ ДАЛЬНЕЙШУЮ ДИСКУССИЮ О БИОГРАФИИ УВАРОВА, ЧАСТЬЮ КОТОРОЙ МОЖНО СЧИТАТЬ И НАСТОЯЩУЮ РЕЦЕНЗИЮ.

\section{NOTES}

1. БИБЛИОГРАФИЮ СМ.: М.Л. МАЙОФИС, М.Б. ВЕЛИЖЕВ, «СЕРГЕЙ СЕМЕНОВИЧ УВАРОВ», РУССКИЕ ПИСАТЕЛИ, 1800-1917: БИОГРАФИЧЕСКИЙ СЛОВАРЬ, Т. 6. М., 2019, С. 368-369. НИЖЕ МЫ ПРИВОДИМ 
СПИСОК КОНЦЕПТУАЛЬНЫХ ПРОЧТЕНИЙ УВАРОВСКОЙ БИОГРАФИИ, КОТОРЫЙ, ВПРОЧЕМ, ОТНЮДЬ НЕ ПРЕТЕНДУЕТ НА ИСЧЕРПАННОСТЬ.

2. C.H. Whittaker, The Origins of Modern Russian Education. An Intellectual Biography of Count Sergei Uvarov, 1786-1855, DeKalb, 1984.

3. Р. ПАЙПС, СЕРГЕЙ СЕМЕНОВИЧ УВАРОВ: ЖИЗНЕОПИСАНИЕ, М., 2013.

4. ПРЕЖДЕ ВСЕГО СМ.: А.Л. ЗОРИН, КОРМЯ ДВУГЛАВОГО ОРЛА... РУССКАЯ ЛИТЕРАТУРА И ГОСУДАРСТВЕННАЯ ИДЕОЛОГИЯ В ПОСЛЕДНЕЙ ТРЕТИ ХVIII - ПЕРВОЙ ТРЕТИ ХІХ ВЕКА М., 2001, С. 339-374.

5. М.Л. МАЙОФИС, ВОЗЗВАНИЕ К ЕВРОПЕ: ЛИТЕРАТУРНОЕ ОБЩЕСТВО «АРЗАМАС» И РОССИЙСКИЙ МОДЕРНИЗАЦИОННЫЙ ПРОЕКТ 1815-1818 ГОДОВ, М., 2008.

6. М.М. ШЕВЧЕНКО, КОНЕЦ ОДНОГО ВЕЛИЧИЯ: ВЛАСТЬ, ОБРАЗОВАНИЕ И ПЕЧАТНОЕ СЛОВО В ИМПЕРАТОРСКОЙ РОССИИ НА ПОРОГЕ ОСВОБОДИТЕЛЬНЫХ РЕФОРМ М., 2003. К ТЕМЕ «УВАРОВКОНСЕРВАТОР» СМ. ТАКЖЕ МАТЕРИАЛЫ СПЕЦИАЛЬНОГО ВЫПУСКА ЖУРНАЛА «ТЕТРАДИ ПО КОНСЕРВАТИЗМУ» (2018, №1), ПОСВЯЩЕННОГО НИКОЛАЕВСКОМУ МИНИСТРУ НАРОДНОГО ПРОСВЕЩЕНИЯ.

7. ФРАНЦУЗСКИЙ ОРИГИНАЛ МЕМУАРОВ СМ.: М.М. ШЕВЧЕНКО, «ОПЫТ АВТОБИОГРАФИИ СЕРГЕЯ УВАРОВА. 1852», ТЕТРАДИ ПО КОНСЕРВАТИЗМУ, 2018, №1, С. 281-297; РУССКИЙ ПЕРЕВОД СМ.: ТАМ ЖЕ, С. 298-318 (ПЕР. М.М. ШЕВЧЕНКО); М.А. ПОЛЯКОВА, УСАДЬБА ПОРЕЧЬЕ УВАРОВЫХ В КУЛЬТУРЕ РОССИИ, М., 2013, С. 138-167 (ПЕР. К.П. АЛЬБОКРИНОВА).

8. СМ. ПИСЬМО БАРТЕНЕВА К П.П. БЕССОНОВУ ОТ 8 ИЮНЯ 1864 Г. ИЗ ПОРЕЧЬЯ: «МЫ ЗДЕСЬ ОКОЛО ТРЕХ НЕДЕЛЬ. РАБОТЫ ЕДВА ЛИ НЕ БОЛЬШЕ, ЧЕМ В МОСКВЕ. ГРАФ УВАРОВ САМ ОЧЕНЬ МНОГО ЗАНИМАЕТСЯ, И ЕГО ПРИМЕР ПОДЗАДОРИВАЕТ. СВЕДЕНИЙ О ПОКОЙНОМ ГРАФЕ МНОЖЕСТВО, И ВЕРОЯТНО Я УСПЕЮ ЗДЕСЬ ТОЛЬКО ПОДГОТОВИТЬ НЕОБХОДИМЫЕ ДАННЫЕ ДЛЯ БИОГРАФИИ. НАПИСАНА ТОЛЬКО ПЕРВАЯ ГЛАВА, О ПРОИСХОЖДЕНИИ И РОДИТЕЛЯХ», ПИСЬМА П.И. БАРТЕНЕВА П.А. БЕССОНОВУ, 1848-1887 ГГ., РОССИЙСКИЙ АРХИВ: ИСТОРИЯ ОТЕЧЕСТВА В СВИДЕТЕЛЬСТВАХ И ДОКУМЕНТАХ ХVIII-ХХ ВВ.: АЛЬМАНАХ, М., 2007, [Т. ХV], С. 285).

9. ПОДРОБНЕЕ СМ.: М.Б. ВЕЛИЖЕВ, «С.С. УВАРОВ В НАЧАЛЕ НИКОЛАЕВСКОГО ЦАРСТВОВАНИЯ: ЗАМЕТКИ К ТЕМЕ», ПУШКИНСКИЕ ЧТЕНИЯ В ТАРТУ 5: ПУШКИНСКАЯ ЭПОХА И РУССКИЙ ЛИТЕРАТУРНЫЙ КАНОН: К 85-ЛЕТИЮ ЛАРИСЫ ИЛЬИНИЧНЫ ВОЛЬПЕРТ, Ч. 2. ТАРТУ, 2011, С. 335-357.

10. ОБЗОР СОДЕРЖАНИЯ СТИХОТВОРНЫХ АЛЬБОМОВ УВАРОВА СМ. В НАШЕМ КОММЕНТАРИИ К ПУБЛИКАЦИИ: Г.М. ДАШЕВСКИЙ, «ГЕДОНИСТИЧЕСКОЕ САМОУБИЙСТВО В РАННЕЙ ЛИРИКЕ ПУШКИНА: ЗАМЕТКИ К ТЕМЕ (ПУБЛ. Н.Н. МАЗУР)», Acta Slavica Estonica XI. ПУШКИНСКИЕ ЧТЕНИЯ В ТАРТУ, 6. ВЫП. 1. ПУШКИН В КРУГУ СОВРЕМЕННИКОВ. ТАРТУ, 2019, С. 42-44.

11. МЫ НАДЕЕМСЯ В БЛИЖАЙШЕМ БУДУЩЕМ ОПУБЛИКОВАТЬ НЕИЗДАННЫЕ ТЕКСТЫ УВАРОВА.

12. СМ. КОММЕНТАРИЙ Е.О. ЛАРИОНОВОЙ В КНИГЕ: Ц.Х. ВИТТЕКЕР, ГРАФ СЕРГЕЙ СЕМЕНОВИЧ УВАРОВ И ЕГО ВРЕМЯ, СПБ., 1999, С. 280.

\section{AUTEURS}

\section{МИХАИЛ ВЕЛИЖЕВ}

ФАКУЛЬТЕТ ГУМАНИТАРНЫХ НАУК НАУЧНО-ИССЛЕДОВАТЕЛЬСКОГО УНИВЕРСИТЕТА ВЫСШАЯ шкОЛА эКОНОМИКИ, МОСКВА 